\title{
Comparing interferon-gamma release assays with tuberculin skin test for identifying latent tuberculosis infection that progresses to active tuberculosis: systematic review and meta-analysis
}

Peter Auguste ${ }^{1 *}$, Alexander Tsertsvadze ${ }^{2}$, Joshua Pink ${ }^{1}$, Rachel Court ${ }^{1}$, Noel McCarthy², Paul Sutcliffe ${ }^{1}$ and Aileen Clarke ${ }^{1}$

\begin{abstract}
Background: Timely and accurate identification of people with latent tuberculosis infection (LTBI) is important for controlling Mycobacterium tuberculosis (TB). There is no gold standard for diagnosis of LTBI. Screening tests such as interferon gamma release assays (IGRAs) and tuberculin skin test (TST) provide indirect and imperfect information. This systematic review compared two types of IGRAs QuantiFERON®-TB Gold In-Tube test (QFT-GIT) and T-SPOT.TB with TST for identification of LTBI by predicting progression to a diagnosis of active TB in three subgroups: children, immunocompromised people, and those recently arrived from countries with high TB burden.

Methods: Cohort studies were eligible for inclusion. We searched MEDLINE, EMBASE, the Cochrane Library and other databases from December 2009 to June 2015. One reviewer screened studies, extracted data, and assessed risk of bias with cross checking by a second reviewer. Strength of association between test results and incidence of TB was summarised using cumulative incidence ratios (CIRs with $95 \% \mathrm{Cls}$ ). Summary effect measures: the ratio of CIRs (R-CIR) with 95\% Cls. R-CIRs, were pooled using a random-effects model. Heterogeneity was assessed using Chi-squared and $\mathrm{I}^{2}$ statistics.

Results: Seventeen studies, mostly of moderate or high risk of bias (five in children, 10 in immunocompromised people, and two in those recently arrived) were included. In children, while in two studies, there was no significant difference between QFT-GIT and TST ( $\geq 5 \mathrm{~mm}$ ) (pooled R-CIR $=1.11,95 \% \mathrm{Cl}: 0.71,1.74$ ), two other studies showed QFT-GIT to outperform TST ( $\geq 10 \mathrm{~mm}$ ) in identifying LTBI. In immunocompromised people, IGRA (T-SPOT.TB) was not significant different from TST $(\geq 10 \mathrm{~mm}$ ) for identifying $L T B I$, (pooled R-CIR $=1.01,95 \% \mathrm{Cl}: 0.65,1.58)$. The forest plot of two studies in recently arrived people from countries with high TB burden demonstrated inconsistent findings (high heterogeneity; $\left.\right|^{2}=92 \%$ ).

Conclusions: Prospective studies comparing IGRA testing against TST on the progression from LTBI to TB were sparse, and these results should be interpreted with caution due to uncertainty, risk of bias, and unexplained heterogeneity. Population-based studies with adequate sample size and follow-up are required to adequately compare the performance of IGRA with TST in people at high risk of TB.
\end{abstract}

Keywords: Systematic review, Latent tuberculosis infection, Interferon gamma release assays, Tuberculin skin test

\footnotetext{
* Correspondence: p.auguste@warwick.ac.uk

'Warwick Evidence, Warwick Medical School, University of Warwick, Coventry

CV4 7AL, UK

Full list of author information is available at the end of the article
} 


\section{Background}

The timely and accurate identification and prophylactic treatment of people with latent tuberculosis infection (LTBI) are important for controlling Mycobacterium tuberculosis (TB) worldwide. Once infected with LTBI, most people remain asymptomatic and are not contagious. However, $5-10 \%$ of those infected may progress to active $\mathrm{TB}$ in their lifetime and become infectious [1]. The risk of progression is higher in younger children [2], people who are immunocompromised or immunosuppressed $[3,4]$, and in people from countries with a high incidence of TB ( $\geq 40$ cases per 100,000) [5].

There is no gold standard for the diagnosis of LTBI. Available screening tests provide indirect information on the presence of LTBI. Historically, the diagnosis of LTBI has relied on the use of the tuberculin skin test (TST) [6]. Recently, interferon gamma release assays (IGRAs) have been developed. These may overcome some of the limitations of TST (e.g., cross-reactivity in Bacilli CalmetteGuerin vaccinated people, error in measuring the size of induration of the skin reaction) and can be used as a replacement or adjunct to the TST. Currently, two IGRAs are commercially available: QuantiFERON-TB Gold Intube (QFT-GIT) (Cellestis Ltd., Carnegie, Australia) and T-SPOT.TB (Oxford Immunotec Ltd, Oxford, UK).

Since the introduction of IGRAs, an increasing number of studies has compared their performance with TST for identification of LTBI. In the absence of a gold standard, these studies have measured a) the association between test results and surrogate measures (e.g., duration or proximity of exposure to an index TB case), b) compared specificity of tests in people at low risk of TB (e.g., healthy people or people from low TB incidence countries) or c) compared sensitivity of tests against cultureconfirmed individuals with $\mathrm{TB}$ [6]. The results from these studies may be biased due to exposure misclassification. Moreover, the findings from studies using the diagnosis of TB as a marker for LTBI may also be biased, given the difference between the two entities.

Other studies have compared the strength of association between IGRA and TST test results in relation to the risk of progression to active TB. The comparison is based on the assumption that people with LTBI are at greater risk of progression to active TB compared to those without it. With this proxy measure, IGRA and TST tests have been compared for their ability to predict progression from LTBI to active TB. For example, two meta-analyses [1, 7] synthesised evidence from primary studies comparing IGRAs to TST using progression to active TB as a proxy for LTBI. Although this approach provides a potentially unbiased estimate of performance, these meta-analyses had methodological limitations. For example, the first meta-analysis included and synthesised studies in which IGRA or TST test positive people were treated with anti-TB prophylactic agents [7]. However evidence suggests that the currently available treatments for LTBI are effective in preventing a reactivation of TB (60-90\%) [5], hence treatments would have had an independent impact on the performance of the IGRA and TST tests. In the second meta-analysis studies of inhouse' assays were included [1]. Little is known about the quality and consistency of these tests across clinical laboratories (UK Standards for Microbiology Investigations) since they are not subject to the regulations of commercially developed tests. Finally, none of the two meta-analyses compared individual IGRAs to TST in predicting risk of progression to active TB separately in children, immunocompromised people, and those who have recently arrived from high TB burden countries.

In this systematic review we aimed to identify, appraise, and synthesise the relevant evidence from longitudinal cohort studies comparing performance of both types of IGRA to TST in identifying LTBI through predicting progression to active TB separately for children, immunocompromised people, and those who have recently arrived from high TB burden countries.

\section{Methods}

This review was conducted as part of a clinical guideline commissioned by the National Institute for Health Research (NIHR) Health Technology Assessment (HTA) Programme (project number 13/178/01) [8].

\section{Inclusion and exclusion criteria}

We included English language reports of head-to-head comparative cohort studies aimed at identifying LTBI which followed-up people to incidence of active TB after testing with IGRAs (QFT-GIT, T-SPOT.TB) and TST separately in children ( $<18$ years), immunocompromised people (e.g., people with HIV, transplant recipients, people receiving or about to start anti-tumour necrosis factor TNF- $\alpha$ treatment), and people arriving from high incidence TB areas (annual incidence $\geq 40$ per 100,000) [5]. We excluded studies of people treated with antituberculosis prophylaxis after testing for LTBI, studies which used 'in-house' assays, and single-arm studies testing people for LTBI with only IGRAs or TST.

\section{Outcomes of interest}

The proportion of people progressing to active TB.

\section{Search strategy}

We searched MEDLINE (Ovid), The Cochrane Library, MEDLINE In-Process Citations and Daily Update (Ovid), EMBASE (Ovid), and Science Citation Index (Web of Knowledge). Searches were limited to English Language studies published between January 2009 and June 2015. 
Electronic searches were supplemented by manually searching reference lists of potentially relevant studies, contacting experts in the field and screening of manufacturers' and other relevant websites. For unpublished studies, we searched specific conference proceedings for the last 5 years. Details of the search strategy can be found in Additional file 1.

\section{Study selection, data extraction, and risk of bias assessment}

Two independent reviewers (AT and PA) screened the titles and abstracts of all identified articles, and afterwards full-texts of potentially relevant articles using pre-piloted forms. One reviewer (PA) extracted relevant data from included studies using a pre-piloted data extraction form. Data extraction was cross-checked by an independent reviewer (AT). Data were collected on author, year, country, and duration of follow-up, population characteristics (age, sex, sub-group), intervention (types of IGRAs), comparator (TST, cut-off values), Bacillus Calmette-Guérin (BCG) status, TB diagnosis, and outcomes (the proportion of people who progressed to active TB). Risk of bias was assessed using the Quality in Prognosis Studies (QUIPS) tool, developed to appraise studies reporting the associations between prognostic factors and health outcomes [9]. The tool addresses the risk of bias for six domains: patient selection/participation, study sample attrition, index test measurement, outcome/construct validity measurement, confounding, and statistical analysis/outcome reporting. Any disagreements at study selection, data extraction, and risk of bias assessment phases of the review were resolved by discussions between the two reviewers or through adjudication of a third independent reviewer.

\section{Data synthesis and analysis}

Given the absence of a gold standard for diagnosing LTBI, the performance of tests was compared using alternative methodology which relies on the validation of test results against a predetermined validity construct (i.e. a proxy for a reference standard) - progression to active TB. For each test (IGRA or TST), the strength of association between test results and incidence of active TB was expressed using cumulative incidence ratios (CIRs; the ratio of active TB incidence in test positives versus $\mathrm{TB}$ incidence in test negatives) with corresponding 95\% CIs. A statistically significant estimate of CIR > 1 would indicate that a test (IGRA or TST) has discriminatory power in predicting occurrence of active TB (i.e. of identifying LTBI). The effect measures comparing IGRAs to TST were summarised as ratios of CIRs (RCIRs) for IGRA vs. TST with 95\% CIs. A statistically significant estimate of R-CIR $>1$ would suggest for example that an IGRA has a better power of predicting the occurrence of active TB (i.e., of identifying LTBI) than TST. Synthesised data were stratified by type of IGRA (QFT-GIT or T-SPOT.TB) and TST threshold $(\geq 5 \mathrm{~mm}$, $\geq 10 \mathrm{~mm}, \geq 15 \mathrm{~mm}$ ). We have not synthesised data from studies of QFT-G because this test is no longer commercially available. We used a random-effects model to pool the summary effect measure (R-CIR) across studies when deemed appropriate and feasible (e.g., no evidence of clinical and methodological heterogeneity, the same cut-off value of TST). We did not pool study results if there was evidence of important clinical or statistical heterogeneity or if data were insufficient. The presence of heterogeneity was judged by visual inspection of forest plots of R-CIRs (and degree of overlap across 95\% CIs), formal statistical tests (Chi-square $<0.10$ and the $\mathrm{I}^{2}$ statistic $>50 \%$ ), or if data permitted a subgroup analysis with respect to a priori defined factors including: BCG vaccination status, risk of bias, TST threshold $(\geq 5 \mathrm{~mm}, \geq$ $10 \mathrm{~mm}, \geq 15 \mathrm{~mm}$ ) and prevalence of TB in country of origin. Publication bias exploration, where data permitted, was planned using asymmetry of contour-enhanced funnel plots from the meta-analyses [10].

\section{Results}

\section{Study identification process}

Of the 7,611 records identified, 515 were selected for full-text examination. Of these, 498 records were excluded. The remaining 17 publications were included in the review [11-27]. Figure 1 shows the study flow with reasons for exclusion depicted in the PRISMA flow diagram [28].

\section{Characteristics of included studies}

Of the 17 included studies, five were conducted in children [11, 13-16], 10 in immunocompromised people $[12,17-25]$, and two studies $[26,27]$ were undertaken in people recently arrived from countries with a high incidence of TB. Most were prospective cohort studies although two [12,18] (both in immunocompromised people) were retrospective cohorts. Further details on baseline characteristics of included studies are provided in Table 1.

\section{Children}

The five studies were undertaken in Germany [11], Turkey [14], Iran [15], South Africa [13] and South Korea [16].

Three studies [11, 13, 16] compared QFT-GIT with TST $(5 \mathrm{~mm} / 10 \mathrm{~mm})$. One study [15] compared QFT-G with TST $(10 \mathrm{~mm})$. The prevalence of BCG vaccination was reported in three studies as ranging from 36 to $94 \%$ $[11,13,16]$. The mean length of follow-up to diagnosis of active TB ranged from 1 year [15] to 4 years [11, 13]. 


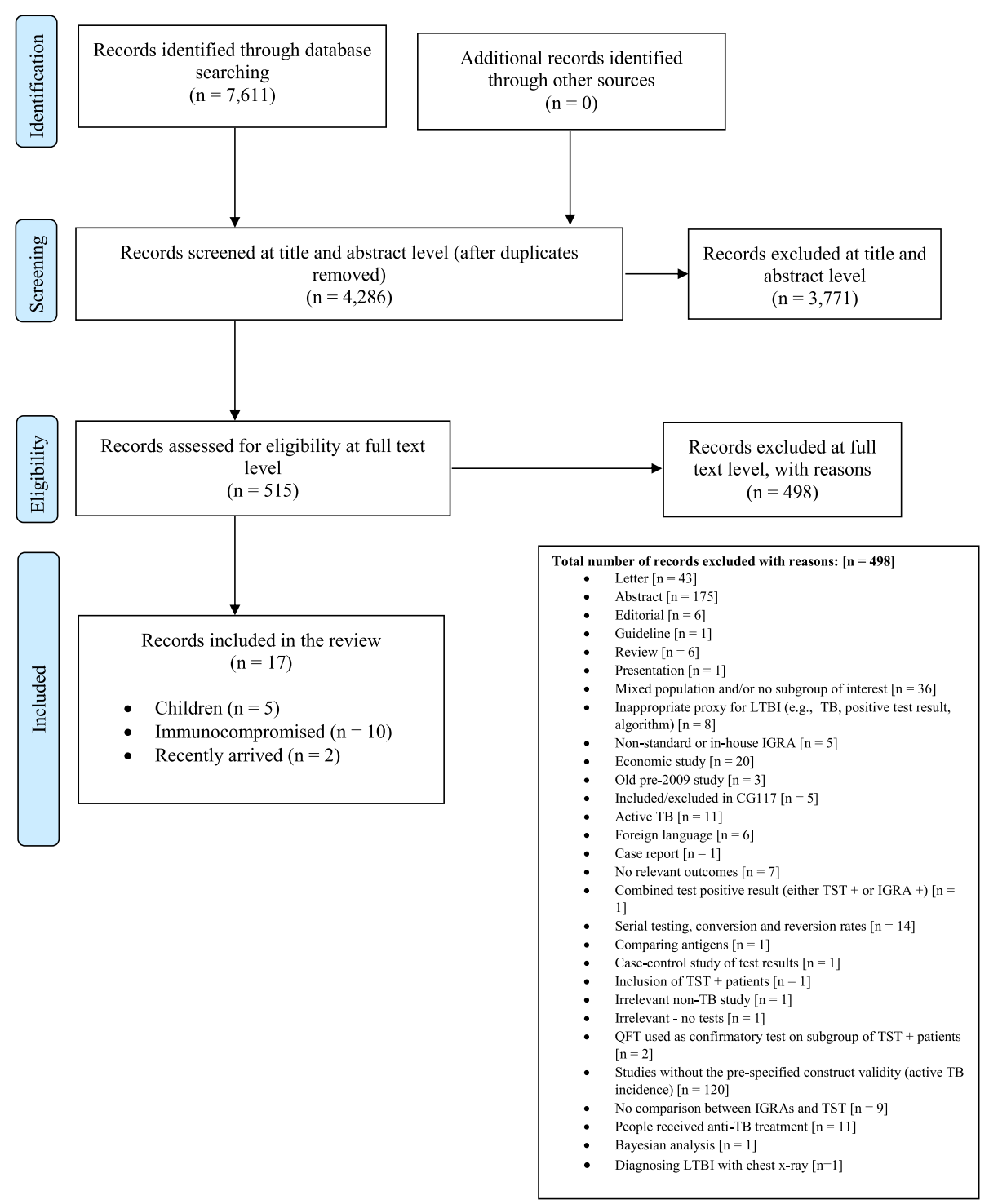

Fig. 1 PRISMA [28] flow diagram

Three studies $[11,13,15]$ clearly stated the method(s) used to diagnose TB.

\section{Immunocompromised people}

Six of the 10 studies were conducted in South Korea and Taiwan [17-19, 21-23], one each in Iran [25], Switzerland [12], and Denmark [20] and the remaining study across various European countries [24].

In two studies, participants were receiving haemodialysis for end-stage renal disease (ESRD) [19, 25]. Two other studies included haematopoietic stem cell transplantation candidates [21] and haematopoietic stem cell transplantation recipients [22]. The remaining six studies included people with 'rheumatic disease' [18], people who had undergone kidney transplantation [17], people living with human immunodeficiency virus (PLWHIV) [12], people being treated for inflammatory arthritis [23], people being treated for sarcoidosis [20], and participants with various conditions and diseases (PLWHIV, chronic renal failure, rheumatoid arthritis, solid-organ transplant or stem-cell transplantation) [24].

Four studies compared T-SPOT.TB to TST $(5 \mathrm{~mm} /$ $10 \mathrm{~mm})$ [12, 17, 19, 25], two studies QFT-G to TST $(10 \mathrm{~mm})$ [19] or TST $(6 \mathrm{~mm} / 12 \mathrm{~mm})$ [20], four studies compared QFT-GIT to either TST $(5 \mathrm{~mm})[18,21]$ or TST $10 \mathrm{~mm} / 15 \mathrm{~mm}[22,23]$. The study undertaken by Sester and colleagues [24] compared three tests (TST measured at $5 \mathrm{~mm}$, QFT-GIT and T-SPOT.TB). The mean follow-up duration across studies ranged from 1.2 to 5 years. Seven studies [17-20, 22-24] reported methods for TB diagnosis. 


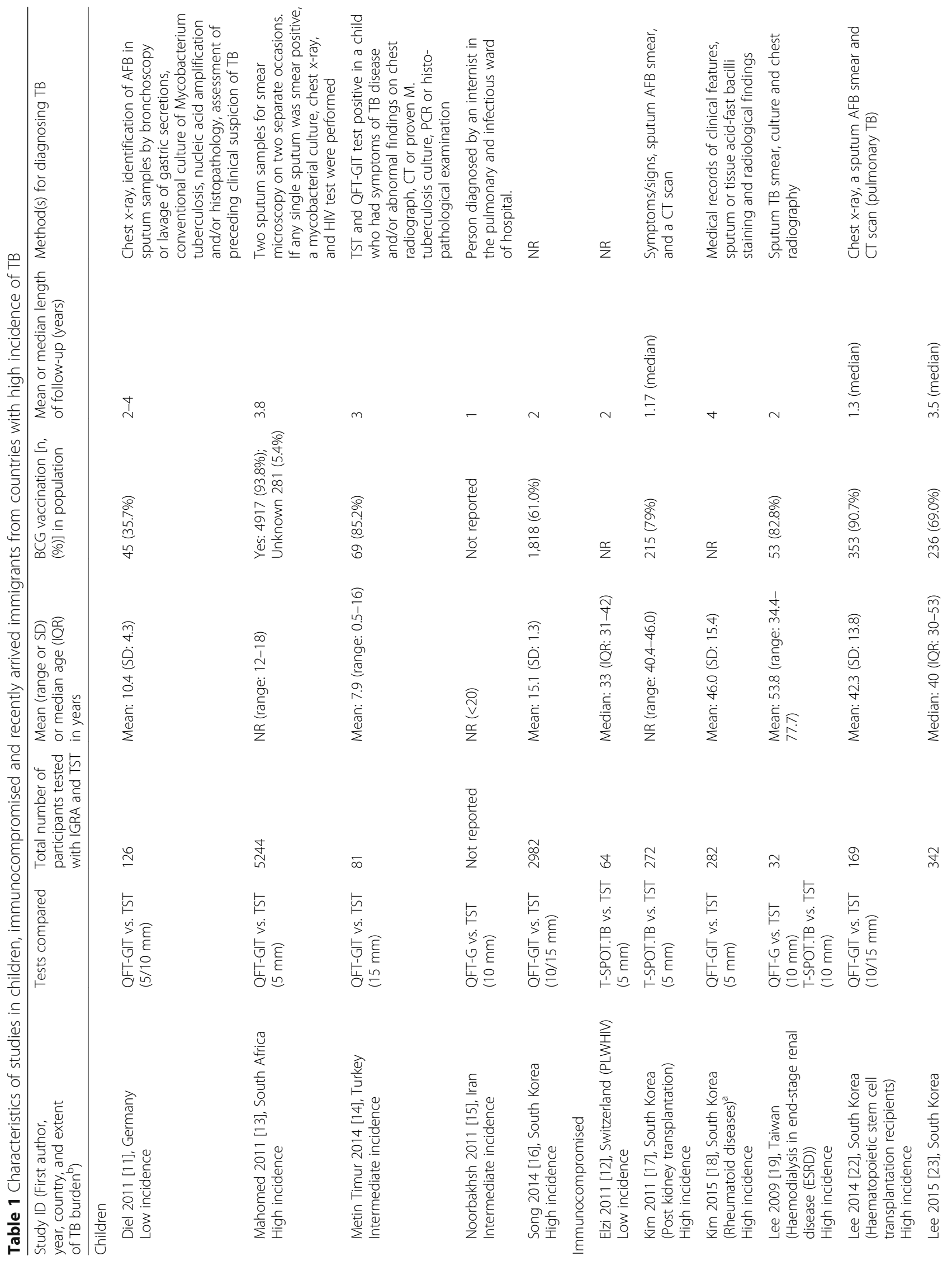




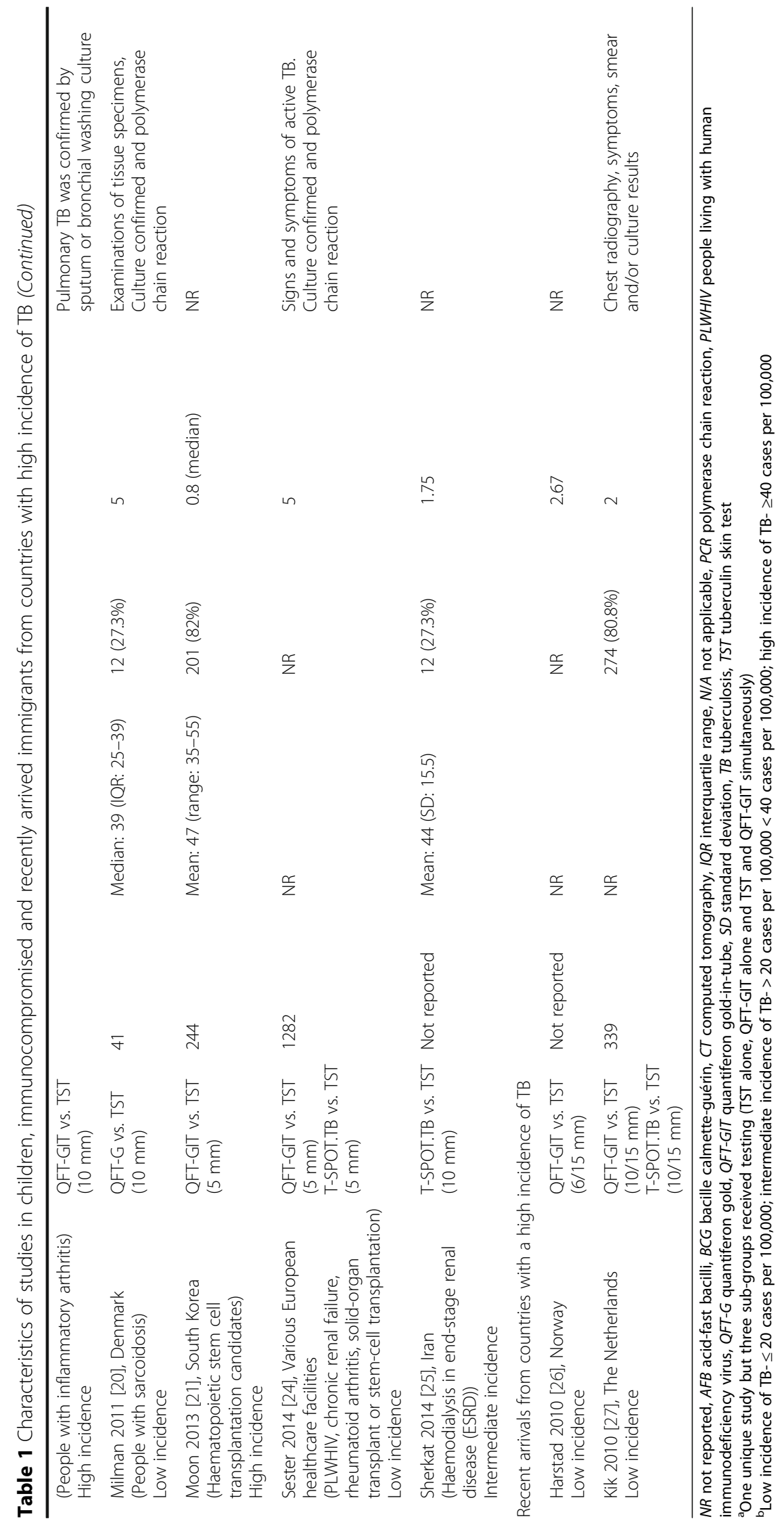




\section{People who recently arrived from countries with high TB incidence}

We identified only two studies [26, 27] conducted in people recently arriving from high TB incidence countries. These studies were undertaken in Norway [26] and the Netherlands [27]. The Harstad et al. study [26] included adult asylum seekers and the Kik et al. [27] study adults who were recently exposed to infectious pulmonary TB. Most of the participants in both studies had arrived from Europe, Africa, and Asia. The studies compared QFT-GIT with TST ( $\geq 6 \mathrm{~mm}$ and $\geq 15 \mathrm{~mm}$ ) [26] and QFT-GIT/T-SPOT.TB with TST $(\geq 10 \mathrm{~mm}$ and $\geq$ $15 \mathrm{~mm}$ ) [27]. The prevalence of BCG vaccination was reported in only one of the studies at $81 \%$ [27]. Mean length of follow-up ranged from 2 years [27] to 3 years [26]. Only one study provided sufficient information on method(s) used to diagnose TB, which included chest radiography, symptoms, smear and/or culture results [27].

\section{Assessment of risk of bias}

The risk of bias by domain and overall is presented in Table 2. In children, two studies $[14,15]$ had a high risk and the remaining three studies a moderate risk of bias
$[11,13,16]$. Most studies had a moderate risk of bias for misclassification of individuals in relation to construct validity groups, since no clear definitions and ascertainment methods were provided $[11,13,15]$. In immunocompromised people, three studies had an overall high $[12,19,25]$ and another three a moderate risk of bias $[21,22,24]$. The remaining four studies had a low overall risk of bias [17, 18, 20, 23]. Five studies [12, 19, 21, 22, 25] had moderate/high risk of bias for the items of study participation, outcome measurement and study confounding.

Of the two studies in a recently arrived people from high TB burden countries, one study had a high overall risk of bias [26] and the other, low risk of bias [27]. In the Harstad study [26], high risk of bias was noted in most of the bias domains (e.g., the study participation, prognostic factor measurement, study confounding, and statistical analysis and reporting domains).

\section{The incidence of active TB following the testing for LTBI by subgroups of interest}

Details on incidence of active TB by LTBI test results are presented for the subgroups of interest in Table 3. IGRAs and TST $(5 \mathrm{~mm})$ were both significantly effective across studies in detecting LTBI for children and

Table 2 Risk of bias in studies of active TB incidence comparing IGRA with TST in children, immunocompromised people and recently arrived people from countries with a high incidence of TB

\begin{tabular}{|c|c|c|c|c|c|c|c|}
\hline First author, Year & $\begin{array}{l}\text { Study } \\
\text { Participation }\end{array}$ & $\begin{array}{l}\text { Study } \\
\text { Attrition }\end{array}$ & $\begin{array}{l}\text { Prognostic Factor } \\
\text { Measurement }\end{array}$ & $\begin{array}{l}\text { Outcome/Construct } \\
\text { Measurement }\end{array}$ & $\begin{array}{l}\text { Study } \\
\text { Confounding }\end{array}$ & $\begin{array}{l}\text { Statistical Analysis } \\
\text { and Reporting }\end{array}$ & Total ROB \\
\hline \multicolumn{8}{|l|}{ Children } \\
\hline Diel, 2011 [11] & Low & Low & Moderate & Moderate & Low & Low & Moderate ROB \\
\hline Mahomed, 2011 [13] & Moderate & Moderate & Moderate & Moderate & High & Low & Moderate ROB \\
\hline Metin Timur 2014 [14] & High & High & Moderate & Moderate & High & High & High ROB \\
\hline Noorbakhsh 2011 [15] & High & High & High & Moderate & High & High & High ROB \\
\hline Song, 2014 [16] & Low & Moderate & Low & High & Moderate & Low & Moderate ROB \\
\hline \multicolumn{8}{|l|}{ Immunocompromised } \\
\hline Elzi, 2011 [12] & High & Low & Low & Moderate & High & Low & High ROB \\
\hline Kim, 2011 [17] & Low & Low & Low & Low & Moderate & Low & Low ROB \\
\hline Kim, 2015 [18] & Low & Low & Low & Low & High & Low & Low ROB \\
\hline Lee, 2009 [19] & High & Low & Low & Moderate & High & Low & High ROB \\
\hline Lee, 2014 [22] & High & Moderate & Moderate & Moderate & Low & Low & Moderate ROB \\
\hline Lee, 2015 [23] & Low & Low & Moderate & Low & High & Low & Low ROB \\
\hline Milman, 2014 [20] & Low & Low & Moderate & Low & Low & Low & Low ROB \\
\hline Moon, 2013 [21] & Moderate & Low & Moderate & Moderate & Moderate & Low & Moderate ROB \\
\hline Sester, 2014 [24] & Low & Low & Moderate & Low & High & Low & Moderate ROB \\
\hline Sherkat, 2014 [25] & High & High & Moderate & High & High & Moderate & High ROB \\
\hline \multicolumn{8}{|c|}{ Recent arrivals from countries with a high incidence of TB } \\
\hline Harstad, $2010[26]$ & High & Low & High & Moderate & High & High & High ROB \\
\hline Kik, 2010 [27] & Low & Low & Low & Low & Low & Low & Low ROB \\
\hline
\end{tabular}


Table 3 Progression to TB following LTBI testing with IGRAs and TST in children, immunocompromised and recently arrived immigrants

\begin{tabular}{|c|c|c|c|c|c|c|}
\hline $\begin{array}{l}\text { Study ID (First } \\
\text { author, year) }\end{array}$ & $\begin{array}{l}\text { Total test } \\
\text { results available }\end{array}$ & $\begin{array}{l}\text { Type of IGRA test } \\
\text { and TST (thresholds) }\end{array}$ & $\begin{array}{l}\text { Number of people } \\
\text { with positive results }\end{array}$ & $\begin{array}{l}\text { Number of people } \\
\text { with negative results }\end{array}$ & $\begin{array}{l}\text { People with test positive results } \\
\text { who progressed to TB (n) }\end{array}$ & $\begin{array}{l}\text { People with test negative results } \\
\text { who progressed to TB (n) }\end{array}$ \\
\hline \multicolumn{7}{|l|}{ Children } \\
\hline \multirow[t]{3}{*}{ Diel, 2011 [11] } & 104 & QFT-GIT & 21 & 83 & 6 & 0 \\
\hline & & TST ( $\geq 5$ mm) & 40 & 64 & 6 & 0 \\
\hline & & TST ( $\geq 10$ mm) & 40 & 64 & 4 & 2 \\
\hline \multirow[t]{2}{*}{ Mahomed, 2011 [13] } & 5244 & QFT-GIT & 2669 & 2575 & 39 & 13 \\
\hline & & TST ( $\geq 5$ mm) & 2894 & 2350 & 40 & 12 \\
\hline \multirow[t]{2}{*}{ Metin Timur, 2014 [14] } & 69 & QFT-GIT & 0 & 69 & 0 & 0 \\
\hline & & TST ( $\geq 15$ mm) & 69 & 0 & 0 & 0 \\
\hline \multirow[t]{2}{*}{ Noorbakhsh, 2011 [15] } & 59 & QFT-G & 18 & 41 & 10 & 0 \\
\hline & 58 & TST $(\geq 10 \mathrm{~mm})$ & 8 & 50 & 3 & 7 \\
\hline \multirow[t]{3}{*}{ Song, 2014 [16] } & 2966 & QFT-GIT & 317 & 2649 & 11 & 12 \\
\hline & 2982 & TST ( $\geq 10 \mathrm{~mm})$ & 663 & 2319 & 13 & 10 \\
\hline & & TST ( $\geq 15$ mm) & 231 & 2751 & 13 & 10 \\
\hline \multicolumn{7}{|l|}{ Immunocompromised } \\
\hline \multirow[t]{2}{*}{ Elzi, 2011 [12] } & 43 & T-SPOT.TB & 25 & 18 & 25 & 18 \\
\hline & 44 & TST ( $\geq 5$ mm) & 22 & 22 & 22 & 22 \\
\hline \multirow[t]{2}{*}{ Kim, 2011 [17] } & 265 & T-SPOT.TB & 89 & 176 & 4 & 0 \\
\hline & 288 & TST ( $\geq 5$ mm) & 26 & 262 & 1 & 3 \\
\hline \multirow[t]{2}{*}{ Kim, $2015^{\mathrm{a}}[18]$} & 282 & QFT-GIT & 7 & 275 & 0 & 1 \\
\hline & 282 & TST ( $\geq 5$ mm) & 12 & 270 & 0 & 1 \\
\hline \multirow[t]{3}{*}{ Lee, 2009 [19] } & 30 & QFT-G & 12 & 18 & 1 & 0 \\
\hline & & T-SPOT.TB & 15 & 17 & 0 & 2 \\
\hline & & TST ( $\geq 10$ mm) & 20 & 12 & 1 & 1 \\
\hline \multirow[t]{3}{*}{ Lee, 2014 [22] } & 159 & QFT-GIT & 26 & 133 & 3 & 2 \\
\hline & 169 & TST ( $\geq 10$ mm) & 19 & 150 & 0 & 5 \\
\hline & & TST ( $\geq 15$ mm) & 12 & 157 & 0 & 5 \\
\hline \multirow[t]{2}{*}{ Lee, 2015 [23] } & 342 & QFT-GIT & 103 & 239 & N/A & 4 \\
\hline & 239 & TST ( $\geq 10$ mm) & 60 & 179 & 2 & 2 \\
\hline \multirow[t]{2}{*}{ Milman, 2011 [20] } & 41 & QFT-G & 0 & 41 & 0 & 0 \\
\hline & 12 & TST ( $\geq 10$ mm) & 0 & 12 & 0 & 0 \\
\hline \multirow[t]{2}{*}{ Moon, 2013 [21] } & 210 & QFT-GIT & 40 & 170 & 1 & 1 \\
\hline & 244 & TST ( $\geq 5$ mm) & 39 & 205 & 0 & 2 \\
\hline \multirow[t]{3}{*}{ Sester, 2014 [24] } & 1238 & QFT-GIT & 159 & 1079 & 3 & 5 \\
\hline & 1217 & T-SPOT.TB & 193 & 1024 & 4 & 6 \\
\hline & 1282 & TST ( $\geq 5$ mm) & 149 & 1133 & 4 & 7 \\
\hline \multirow[t]{2}{*}{ Sherkat, 2014 [25] } & 44 & T-SPOT.TB & 6 & 38 & 1 & 0 \\
\hline & & TST ( $\geq 10$ mm) & 8 & 36 & 1 & 0 \\
\hline \multicolumn{7}{|c|}{ Recent arrivals from countries with a high incidence of TB } \\
\hline \multirow[t]{3}{*}{ Harstad, 2010 [26] } & 815 & QFT-GIT & 238 & 577 & 8 & 1 \\
\hline & & TST ( $\geq 6$ mm) & 415 & 395 & 8 & 1 \\
\hline & 813 & TST ( $\geq 15$ mm) & 121 & 692 & 3 & 6 \\
\hline \multirow[t]{4}{*}{ Kik, 2010 [27] } & 327 & QFT-GIT & 178 & 149 & 5 & 3 \\
\hline & 299 & T-SPOT.TB & 181 & 118 & 6 & 2 \\
\hline & 339 & TST $(\geq 10 \mathrm{~mm})$ & 288 & 51 & 9 & 0 \\
\hline & 322 & TST ( $\geq 15$ mm) & 184 & 138 & 7 & 1 \\
\hline
\end{tabular}


immunocompromised people. Among immunocompromised people and those recently arrived from high incidence countries findings were not statistically significant for TST $(10 \mathrm{~mm})$ in predicting progression to active TB. Among recent arrivals, T-SPOT.TB test results were also not statistically significantly associated with progression to active TB.

\section{Children}

QFT-GIT

Fifty-six of the 3007 (1.86\%) QFT-GIT-positive children (4 studies $[11,13,14,16])$ progressed to active TB compared with 25 of the $5376(0.46 \%)$ QFT-GIT-negative children (overall crude CIR for QFT-GIT: 1.86/0.46= 4.01, 95\% CI: 2.51, 6.40).

\section{TST $(5 \mathrm{~mm})$}

Forty-six of the 2934 (1.56\%) TST ( $\geq 5 \mathrm{~mm}$ )-positive children (2 studies [11, 13]) progressed to TB compared with 12 of $2414(0.49 \%)$ TST ( $<5 \mathrm{~mm})$-negative children of whom only $12(0.49 \%)$ progressed to active TB (overall crude CIR for TST-5 mm: $1.57 / 0.50=3.14,95 \%$ CI: $1.68,5.94)$.

\section{TST $(10 \mathrm{~mm})$}

Twenty of the $711(2.81 \%)$ TST ( $\geq 10 \mathrm{~mm})$-positive children (3 studies $[11,15,16])$ progressed to TB compared with 19 of the $2433(0.78 \%)$ TST (<10 mm)-negative children (crude CIR for TST-10 mm: $2.81 / 0.78=3.60$, 95\% CI: 1.93, 6.71).

\section{Immunocompromised}

\section{IGRAs (QFT-GIT and T-SPOT.TB)}

In the immunocompromised population (4 studies $[18,21,22,24])$, seven of the $232(3.02 \%)$ QFT-GIT positive people progressed to active TB compared with 13 of the $1999(0.65 \%)$ QFT-GIT that tested negative (crude CIR for QFT-GIT: 3.02/0.65 = 4.65, 95\% CI: $1.87,11.51) .34$ of the $328(10.37 \%)$ T-SPOT.TB positive people (5 studies [12, 17, 19, 24, 25]) progressed to TB compared with 26 of the 1273 (2.04\%) T-SPOT.TB that tested negative (crude CIR for T-SPOT.TB: 10.37/ $2.04=5.08$, 95\% CI: 3.09, 8.33).

\section{TST $(10 \mathrm{~mm})$}

Four of the 107 (3.74\%) people with TST $(\geq 10 \mathrm{~mm})$ (5 studies $[19,20,22,23,25])$ progressed to TB compared with eight of $389(2.06 \%)$ with TST $(<10 \mathrm{~mm})$ (crude CIR for TST-10 mm: 3.74/2.06 $=1.82,95 \% \mathrm{CI}$ : $0.58,5.92)$.
Recent arrivals from countries with a high incidence of TB IGRAs (QFT-GIT and T-SPOT.TB)

Across two studies [26, 27], 13 of 416 (3.13\%) recent arrivals who tested positive with QFT-GIT progressed to TB compared to four of $726(0.55 \%)$ that tested negative (crude CIR for QFT-GIT: 3.13/0.55 = 5.69, 95\% CI: 1.86, 17.28). Six of 181 (3.31\%) people who tested positive with T-SPOT.TB progressed to TB compared to two of 118 (1.69\%) that tested negative (crude CIR for TSPOT.TB: $3.31 / 1.69=1.96$, 95\% CI: 0.40, 9.53).

\section{TST ( $\geq 6 \mathrm{~mm}$ or $\geq 10 \mathrm{~mm}$ )}

In one study [26] TST ( $\geq 6 \mathrm{~mm}$ ) was used as the threshold for a positive test. Results showed that eight of 415 (1.93\%) people who tested positive progressed to TB compared with one of the $395(0.25 \%)$ people who tested negative (crude CIR for TST-6 mm: 1.93/0.25 =7.72, 95\% CI: $0.96,60.59)$. In the other study [27], 9 of the 288 (3.12\%) people with a TST $(\geq 10 \mathrm{~mm})$ progressed to $\mathrm{TB}$ as compared to none of the $51(0 \%)$ people that tested negative (crude CIR for TST-10 mm: 3.42, 95\% CI: $0.20,57.83)$.

\section{Comparative performance of tests for identifying latent tuberculosis infection \\ Children}

QFT-GIT vs. TST ( $\geq 5 \mathrm{~mm}$ ) Only two studies were eligible for pooling R-CIRs and 95\% CIs to compare QFT-GIT and TST ( $\geq 5 \mathrm{~mm})[11,13]$. The meta-analytic estimate was not statistically significant between QFTGIT and TST $(\geq 5 \mathrm{~mm}$ ) for identifying LTBI (Fig. 2a; pooled CIR $=1.11,95 \%$ CI: $0.71,1.74)$.

QFT-GIT vs. TST $(\geq \mathbf{1 0} \mathbf{~ m m})$ The individual results from two studies tended to favour IGRA (QFT-GIT) to TST ( $\geq 10 \mathrm{~mm}$ ) [11, 16] (Fig. 2b). We did not pool the RCIRs due to significant heterogeneity across the estimates of these studies $\left(p=0.01, \mathrm{I}^{2}=83 \%\right)$. Both studies were at moderate risk of bias, therefore the risk of bias is less likely to explain this heterogeneity. One potential source of heterogeneity may have been the difference in the burden of TB incidence between the two studies. Specifically, the study which showed non-significant difference between IGRA and TST [16] was conducted in a high incidence area (South Korea) as opposed to the study by Diel et al. (2011) [11], which was conducted in low TB incidence area (Germany). There has been evidence showing reduced sensitivity and specificity of IGRAs in high compared to low TB burden areas, where the former is represented by high BCG vaccination rates given at birth [29-33]. 


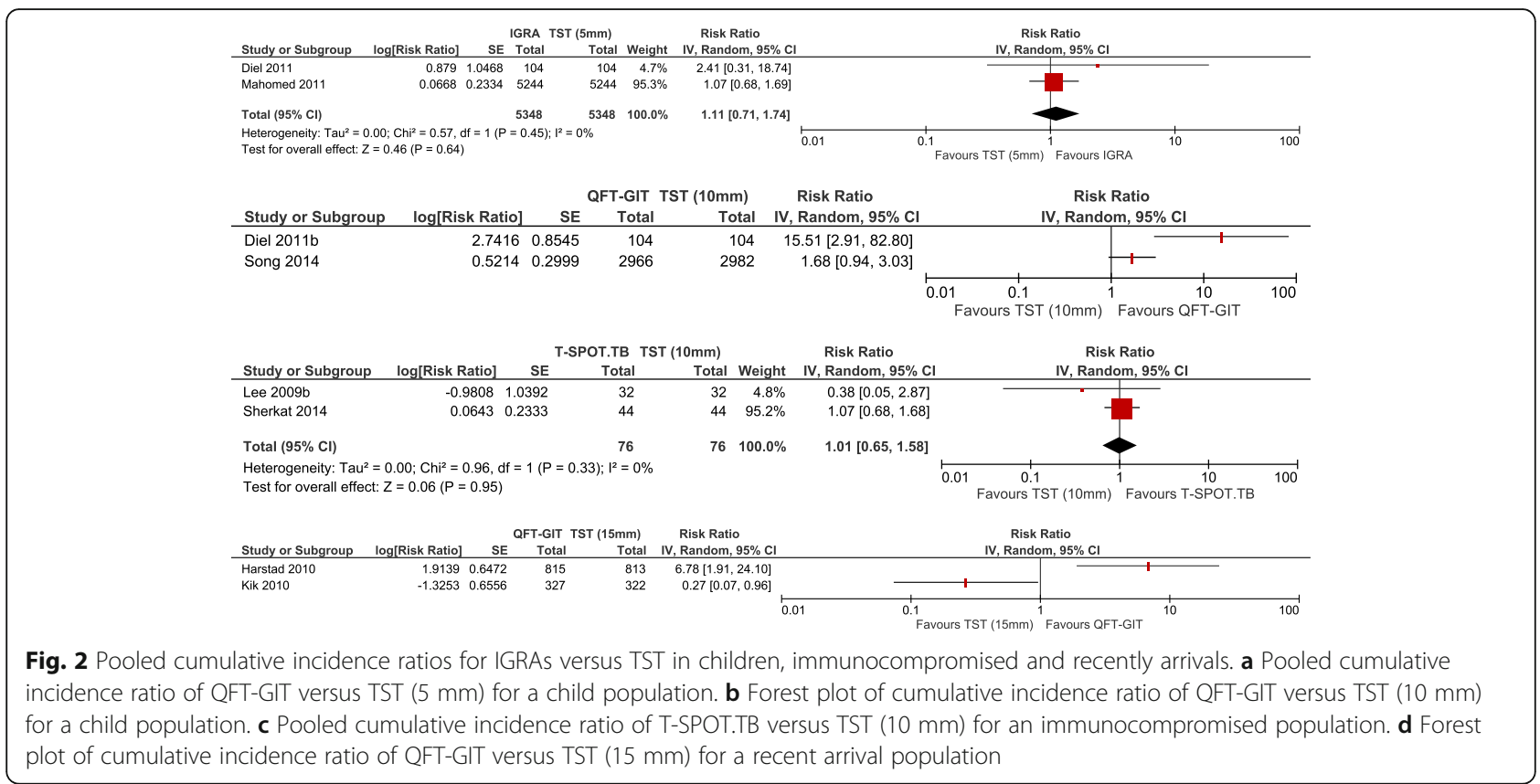

\section{Immunocompromised people}

\section{T-SPOT.TB vs. TST $(\geq 10 \mathrm{~mm})$}

The R-CIRs were pooled across two studies that included an ESRD population (Fig. 2c; pooled R-CIR = 1.01, 95\% CI: $0.65,1.58)[19,25]$. The meta-analytic estimate comparing the performance between IGRA (T-SPOT.TB) and TST ( $\geq 10 \mathrm{~mm})$ was not statistically significant. The corresponding R-CIRs for individual studies were also non-significant: 0.38 (95\% CI: 0.05, 2.87) [19] and 1.07 (95\% CI: 0.68, 1.68) [25]. We did not pool the study estimates across different immunocompromised populations due to clinical heterogeneity.

\section{People who arrived recently from countries of high TB burden QFT-GIT vs. TST ( $\geq 15 \mathrm{~mm}$ )}

Two studies compared QFT-GIT to TST ( $\geq 15 \mathrm{~mm})$ for this population [26, 27]. As Fig. 2d suggests, in the Harstad et al. study [26], QFT-GIT was in favour over TST $(\mathrm{R}-\mathrm{CIR}=6.78,95 \% \mathrm{CI}: 1.91,24.10)$. In contrast, in the Kik et al. study [27], TST was in favour over QFT-GIT ( $\mathrm{R}-\mathrm{CIR}=0.27,95 \% \mathrm{CI}: 0.07,0.96)$. The R-CIR estimates were not pooled due to significant heterogeneity arising from the opposing findings (Fig. $2 \mathrm{~d}$; $p<0.01, \mathrm{I}^{2}=92 \%$ ). The a priori defined factors (BCG vaccination status, TST threshold, risk of bias, and prevalence of TB in country of origin) could not readily explain this inconsistency. Note that the two studies differed in the study populations of asylum seekers [26] vs. immigrants with known contact with an index case [27]. Moreover, the Kik study [27] excluded contacts with TST $<5 \mathrm{~mm}$ which may have influenced test accuracy parameter estimates.

\section{Discussion}

This systematic review compared the performance of IGRAs with TST for identifying LTBI in terms of predicting progression to active TB in children, immunocompromised people and people who had recently arrived from high TB burden countries. There was limited evidence; mostly from studies with moderate to high risk of bias making it difficult to draw definitive conclusions. There was largely consistent evidence in favour of each test predicting progression to active TB, but there was evidence demonstrating that one test outperformed others. Even within the well-defined population categories of this study, there was a great deal of heterogeneity across the R-CIR effect estimates comparing IGRAs to TST, thereby rendering results inconclusive.

There was no evidence indicating that QFT-GIT was better or worse than TST $(5 \mathrm{~mm})$ in detecting LTBI in children. This should not be interpreted as the absence of difference, since the $95 \%$ CIs were wide enough to cover differences of at least moderate size in each direction either favouring IGRA or TST. When QFT-GIT was compared with TST $(10 \mathrm{~mm})$, the individual study estimates tended to favour QFT-GIT over TST, but there was still strong heterogeneity across the studies. One study [16] showed a non-significant difference between QFT-GIT and TST $(10 \mathrm{~mm})$ in a high TB burden setting and the other [11] favoured QFT-GIT over TST $(10 \mathrm{~mm})$ in a low TB burden setting. This observation is consistent with a growing body of evidence showing a reduced sensitivity and specificity of IGRAs in high compared with low TB burden areas, the former represented mostly by developing countries [29-33]. This 
heterogeneity in test performance might be explained by a number of factors relevant to these high TB burden settings for example BCG vaccination is frequently given at birth or there may be a higher frequency of exposure to MTB, different TB transmission dynamics, malnutrition, comorbidity, co-infection with HIV, exposure to non-tuberculous mycobacterium (NTMs) or helminthic infection [32-34].

Similarly, there was no evidence indicating that $\mathrm{T}$ SPOT.TB was better or worse than TST $(10 \mathrm{~mm})$ in detecting LTBI in immunocompromised people. Again, 95\% CIs were compatible with a wide range of values of moderate size in both directions.

The findings in two meta-analysed studies of recently arrived populations from high TB burden areas were in opposite direction. Specifically, one study [27] demonstrated that TST $(15 \mathrm{~mm})$ outperformed QFT-GIT, while the other study [26] showed the opposite. The a priori defined factors (TST threshold, BCG vaccination, risk of bias and TB burden) could not readily explain the inconsistency between these study findings. Other factors, such as inclusion criteria for study population could have contributed to this difference. For example, one study included asylum seekers [26] as opposed to the other study which included immigrants who had contacts with an index case [27]. In addition, the Kik study excluded contacts with TST $<5 \mathrm{~mm}$ [27].

Despite the extensive research in this area, limited evidence is available on progression to TB in untreated populations following testing with commercial IGRAs/ TST. This is likely to be a reflection of the standard of care in high-income countries which is to offer antituberculous treatment to people who test positive. Moreover, some evidence has indicated that there exists variability in TB diagnosis across countries and studies, which further complicates the comparison of diagnostic accuracy of TB detection tests $[35,36]$.

The main strength of this systematic review is that it synthesises the available evidence on progression to $\mathrm{TB}$ in people who have not received anti-TB treatment for LTBI. Moreover, in this review we have evaluated and compared the performance of IGRA and TST tests separately in subgroups of children, immunocompromised, or recently arrived people from countries with high TB burden.

We identified two $[1,7]$ systematic reviews and metaanalyses assessing IGRAs for predicting the incidence of TB. The first review [1] included studies which used inhouse' assays to diagnose LTBI, but little is known about the quality and consistency of these tests across the clinical laboratories (UK Standards for Microbiology Investigations). In addition, people who had indeterminate results at baseline and progressed to TB were assumed to have a negative result. Using this method would decrease the sensitivity and increase the specificity of the test. The second review [7] included studies where people received anti-TB preventative treatment. These studies may be biased as this therapy will decrease the number of people progressing to $\mathrm{TB}$, underestimating the magnitude of the effect estimate for any given test. Since progression to $\mathrm{TB}$ is being used as a reference standard, this will have an impact on the predictive values or sensitivity and specificity of the test. In our review we included only studies in which people were not treated with anti-TB prophylactic treatment and were followed-up to identify progression of TB.

This review has limitations. First, we excluded studies on incidence of TB following serial testing with IGRAs/ TST. If some time has passed since a person becomes infected with $M$. bacterium they may have a negative TST result on initial testing. However, on subsequent TST an individual may have a positive reaction because the initial test stimulates their ability to react to the test. This is commonly referred to as the 'booster phenomenon.' Unlike TST, the use of IGRAs in serial testing does not lead to a 'booster phenomenon.' Despite this, studies using IGRAs to assess reproducibility can potentially lead to conversion/reversion of test results, and this can alter the clinical decision on whether people should be treated for LTBI. Second, included studies did not stratify results by BCG status, so we were unable to present information on people with or without BCG vaccination who tested on IGRA/TST and further developed TB. Likewise, due to sparse data in our meta-analyses (maximum of only two studies pooled), we were unable to construct funnel plots to investigate the effects of publication bias.

More large prospective longitudinal studies or trials comparing head-to-head IGRAs versus TST in untreated populations would help to elucidate the relative merits of IGRA and TST tests in identifying LTBI among different population subgroups. We are aware of one study, the UK Prognostic Evaluation of Diagnostic IGRA Consortium (PREDICT) [37], which will add to existing knowledge as soon as information becomes available. However, there may not be many others given the increasing likelihood of treatment for those testing positively.

Given our findings that tests work but that there is a lack of evidence on which works best, policy makers and those selecting tests should consider practical issues such as the patient population, the availability of tests, and the patient acceptability of the tests [38]. More specifically, the knowledge of sensitivity and specificity of each IGRA and TST for identifying LTBI would also be advantageous. Should decision-makers decide to test, sensitivity and specificity estimates would provide valuable information on the cost-effectiveness of strategies to identify LTBI which progresses to TB in these populations. 


\section{Conclusions}

Longitudinal studies exploring progression rates from LTBI to active TB in children, immunocompromised people, and those recently arrived from areas of high TB burden are sparse. The pooled risk ratios in our analyses did not allow identification of superiority or of non-inferiority of the different tests investigated. Our findings are based on a limited number of studies comparing IGRAs with TST, and the results should be interpreted with caution due to uncertainty, risk of bias, and heterogeneity. Prospective populationbased studies or trials with an adequate sample size and follow-up should be conducted in people who are considered to be at high risk for TB. These studies should employ standard diagnostic methodology and criteria for ascertaining incident cases of active TB. However, there may be difficulties in conducting such studies due to the increasing use of treatment for those who test positive.

\section{Additional file}

Additional file 1: An example of the search strategy used to identify relevant papers. (DOCX $16 \mathrm{~kb}$ )

\begin{abstract}
Abbreviations
BCG: Bacillus Calmette-Guérin; Cl: Confidence intervals; CIR: Cumulative incidence ratio; ESRD: End-stage renal disease; HTA: Health technology assessment; IGRA: Interferon gamma release assay; LTBI: Latent tuberculosis infection; NIHR: National institute for Health Research; NTM: Non-tuberculous mycobacterium; PICO: Population, intervention, comparator and outcome; PLWHIV: People living with human immunodeficiency virus; PRISMA: Preferred reporting items for systematic reviews and meta-analyses; QFT-G: QuantiFERON-TB Gold; QFT-GIT: QuantiFERON ${ }^{\oplus}$-TB Gold In-Tube test; QUIPS: Quality in prognosis studies; R-CIR: Ratio of cumulative incidence ratio; ROB: Risk of bias; TB: Mycobacterium Tuberculosis; TNF: Tumour necrosis factor; TST: Tuberculin skin test
\end{abstract}

\section{Acknowledgements}

This project was funded by the by National Institute for Health Research Health Technology Assessment Programme (13/178/01) and is published in full in the Health Technology Assessment journal series. The views and opinions expressed are those of the authors and do not necessarily reflect those of the Department of Health.

\section{Funding}

This systematic review was funded by the National Institute of Health Research Health Technology Assessment Programme (NIHR HTA) (13/178/01).

\section{Availability of data and materials}

Data available on request from the authors.

\section{Authors' contributions}

PA and AT designed and JP and AT supervised the systematic review. The systematic searches were performed by RC with input from PA and AT. NM provided support on the statistical analyses. PA and AT prepared the manuscript as lead writers. AC and PS co-ordinated the systematic review. All authors read and approved the final manuscript.

\section{Competing interests}

AC is Professor of Public Health \& Health Services Research, Warwick Medical. School, University of Warwick, UK and is a member of the NIHR HTA and EME Editorial board. AC is also supported by the NIHR Collaboration for Leadership in Applied Health Research and Care (CLAHRC) West Midlands at University Hospitals Birmingham NHS Foundation Trust.

\section{Consent of publication}

Not applicable.

Ethics approval and consent to participate Not applicable.

\section{Author details}

${ }^{1}$ Warwick Evidence, Warwick Medical School, University of Warwick, Coventry CV4 7AL, UK. 'Evidence in Communicable Disease Epidemiology and Control, Health Sciences, Warwick Medical School, University of Warwick, Coventry, UK.

Received: 8 September 2016 Accepted: 1 March 2017

Published online: 09 March 2017

\section{References}

1. Diel R, Loddenkemper R, Nienhaus A. Predictive value of interferon- release assays and tuberculin skin testing for progression from latent TB infection to disease state: a meta-analysis. Chest. 2012;142(1):63-75.

2. Kasprowicz VO, Churchyard G, Lawn SD, Squire SB, Lalvani A. Diagnosing latent tuberculosis in high-risk individuals: rising to the challenge in highburden areas. J Infect Dis. 2011;204 Suppl 4:S1168-78.

3. Santin M, Munoz L, Rigau D. Interferon- release assays for the diagnosis of tuberculosis and tuberculosis infection in HIV-infected adults: a systematic review and meta-analysis. PLoS ONE. 2012;7(3), e32482.

4. Chkhartishvili N, Kempker RR, Dvali N, Abashidze L, Sharavdze L, Gabunia P, Blumberg HM, Del Rio C, Tsertsvadze T. Poor agreement between interferon-gamma release assays and the tuberculin skin test among HIVinfected individuals in the country of Georgia. BMC Infect Dis. 2013;13:513.

5. Public Health England. World Health Organization (WHO) estimates of tuberculosis incidence by rate, 2012 (sorted by rate). 2014 [cited; Available from: http://www.hpa.org.uk/web/HPAweb\&HPAwebStandard/HPAweb_C/ 1195733837507

6. Trajman A, Steffen RE, Menzies D. Interferon-Gamma Release Assays versus Tuberculin Skin Testing for the Diagnosis of Latent Tuberculosis Infection: An Overview of the Evidence. Pulm Med. 2013;2013:601737.

7. Rangaka MX, Wilkinson KA, Glynn JR, Ling D, Menzies D, MwansaKambafwile J, Fielding K, Wilkinson RJ, Pai M. Predictive value of interferongamma release assays for incident active tuberculosis: a systematic review and meta-analysis. Lancet Infect Dis. 2012;12(1):45-55.

8. Auguste P, Tsertsvadze A, Pink J, Court R, Seedat F, Gurung T, Freeman K, Taylor-Phillips S, Walker C, Madan J, Kandala NB, Clarke A, Sutcliffe P. Accurate diagnosis of latent tuberculosis in children, people who are immunocompromised or at risk from immunosuppression and recent arrivals from countries with a high incidence of tuberculosis: systematic review and economic evaluation. Health Technol Assess. 2016;20:38.

9. Hayden JA, van der Windt DA, Cartwright $\mathrm{J}$, Cote P, Bombardier C. Assessing bias in studies of prognostic factors. Ann Intern Med. 2013;158(4):280-6.

10. Peters JL, Sutton AJ, Jones DR, Abrams KR, Rushton L. Contour-enhanced meta-analysis funnel plots help distinguish publication bias from other causes of asymmetry. J Clin Epidemiol. 2008;61(10):991-6.

11. Diel R, Loddenkemper R, Niemann S, Meywald-Walter K, Nienhaus A. Negative and positive predictive value of a whole-blood interferon- release assay for developing active tuberculosis: an update. Am J Respir Crit Care Med. 2011;183(1):88-95.

12. Elzi L, Steffen I, Furrer H, Fehr J, Cavassini M, Hirschel B, Hoffmann M, Bernasconi E, Bassetti S, Battegay M. Improved sensitivity of an interferongamma release assay (T-SPOT.TB) in combination with tuberculin skin test for the diagnosis of latent tuberculosis in the presence of HIV co-infection. BMC Infect Dis. 2011;11:319.

13. Mahomed H, Hawkridge T, Verver S, Abrahams D, Geiter L, Hatherill M, Ehrlich R, Hanekom WA, Hussey GD. The tuberculin skin test versus QuantiFERON TB Gold in predicting tuberculosis disease in an adolescent cohort study in South Africa. PLoS ONE. 2011;6(3):e17984. Erratum appears in PLoS One. 2011;6(6). doi:10.1371/annotation/b371be66-de38-4ed6-b440d27c9d7e552c.

14. Metin Timur O, Tanir G, Oz FN, Bayhan Gl, Aydin Teke T, Tuygun N. Comparison of QuantiFERON-TB gold in-tube test with tuberculin skin test in children who had no contact with active tuberculosis case. Tuberkuloz ve Toraks. 2014;62(2):116-21. 
15. Noorbakhsh S, Mousavi J, Barati M, Shamshiri AR, Shekarabi M, Tabatabaei A, Soleimani G. Evaluation of an interferon-gamma release assay in young contacts of active tuberculosis cases. East Mediterr Health J. 2011;17(9):714-8.

16. Song SE, Yang J, Lee KS, Kim H, Kim YM, Kim S, Park MS, Oh SY, Lee JB, Lee E, Park SH, Kim HJ. Comparison of the tuberculin skin test and interferon gamma release assay for the screening of tuberculosis in adolescents in close contact with tuberculosis TB patients. PLoS ONE. 2014;9(7), e100267.

17. Kim SH, Lee SO, Park JB, Park IA, Park SJ, Yun SC, Jung JH, Kim YH, Kim SC, Choi SH, Jeong JY, Kim YS, Woo JH, Park SK, Park JS, Han DJ. A prospective longitudinal study evaluating the usefulness of a T-cellbased assay for latent tuberculosis infection in kidney transplant recipients. Am J Transplant. 2011;11(9):1927-35. Erratum appears in Am J Transplant. 2011 Nov;11(11):2541.

18. Kim JH, Won S, Choi CB, Sung YK, Song GG, Bae SC. Evaluation of the usefulness of interferon-gamma release assays and the tuberculin skin test for the detection of latent Mycobacterium tuberculosis infections in Korean rheumatic patients who are candidates for biologic agents. Int J Rheum Dis. 2015;18(3):315-22.

19. Lee SSJ, Chou KJ, Su IJ, Chen YS, Fang HC, Huang TS, Tsai HC, Wann SR, Lin $\mathrm{HH}$, Liu YC. High prevalence of latent tuberculosis infection in patients in end-stage renal disease on hemodialysis: Comparison of quantiFERON-TB GOLD, ELISPOT, and tuberculin skin test. Infection. 2009;37(2):96-102.

20. Milman N, Soborg B, Svendsen CB, Andersen AB. Quantiferon test for tuberculosis screening in sarcoidosis patients. Scand J Infect Dis. 2011;43(9):728-35.

21. Moon SM, Lee SO, Choi SH, Kim YS, Woo JH, Yoon DH, Suh C, Kim DY, Lee $\mathrm{JH}$, Lee JH, Lee KH, Kim SH. Comparison of the QuantiFERON-TB Gold InTube test with the tuberculin skin test for detecting latent tuberculosis infection prior to hematopoietic stem cell transplantation. Transpl Infect Dis. 2013;15(1):104-9.

22. Lee YM, Lee SO, Choi SH, Kim YS, Woo JH, Kim DY, Lee JH, Lee KH, Kim SH. A prospective longitudinal study evaluating the usefulness of the interferongamma releasing assay for predicting active tuberculosis in allogeneic hematopoietic stem cell transplant recipients. J Infect. 2014;69(2):165-73.

23. Lee $H$, Park HY, Jeon $\mathrm{K}$, Jeong BH, Hwang JW, Lee J, Cha HS, Koh EM, Kang ES, Koh WJ. QuantiFERON-TB gold in-tube assay for screening arthritis patients for latent tuberculosis infection before starting anti-tumor necrosis factor treatment. PLOS ONE. 2015;10:3.

24. Sester M, Van Leth F, Bruchfeld J, Bumbacea D, Cirillo DM, Dilektasli AG, Dominguez J, Duarte R, Ernst M, Eyuboglu FO, Gerogianni I, Girardi E, Goletti D, Janssens JP, Julander I, Lange B, Latorre I, Losi M, Markova R, Matteelli A, Milburn H, Ravn P, Scholman T, Soccal PM, Straub M, Wagner D, Wolf T, Yalcin A, Lange C. Tbnet. Risk assessment of tuberculosis in immunocompromised patients. A TBNET study. Am J Respir Crit Care Med. 2014;190(10):1168-76.

25. Sherkat R, Yaran M, Shoaie P, Mortazavi M, Shahidi S, Hamidi H, Seirafian S, Taheri S, Farajzadegan Z, Rostami S. Concordance of the tuberculin skin test and T-SPOT ().TB test results in kidney transplant candidates. J Res Med Sci. 2014;19 Suppl 1:S26-9.

26. Harstad I, Heldal E, Steinshamn SL, Garasen H, Winje BA, Jacobsen GW Screening and treatment of latent tuberculosis in a cohort of asylum seekers in Norway. Scandinavian Journal of Public Health. 2010;38(3):275-82.

27. Kik SV, Franken WP, Mensen M, Cobelens FG, Kamphorst M, Arend SM, Erkens C, Gebhard A, Borgdorff MW, Verver S. Predictive value for progression to tuberculosis by IGRA and TST in immigrant contacts. Eur Respir J. 2010;35(6):1346-53.

28. Moher D, Liberati A, Tetzlaff J, Altman DG. Preferred reporting items for systematic reviews and meta-analyses: the PRISMA statement. Ann Intern Med. 2009;151(4):264-9. W264.

29. Dinnes J, Deeks J, Kunst H, Gibson A, Cummins E, Waugh N, Drobniewski F, Lalvani A. A systematic review of rapid diagnostic tests for the detection of tuberculosis infection. Health Technol Assess. 2007;11(3):1-196.

30. Machingaidze S, Wiysonge CS, Gonzalez-Angulo Y, Hatherill M, Moyo S, Hanekom W, Mahomed $\mathrm{H}$. The utility of an interferon gamma release assay for diagnosis of latent tuberculosis infection and disease in children: a systematic review and meta-analysis. Pediatr Infect Dis J. 2011;30(8):694-700.

31. Pai M, Zwerling A, Menzies D. Systematic review: T-cell-based assays for the diagnosis of latent tuberculosis infection: an update. Ann Intern Med. 2008; 149(3):177-84.

32. Dheda K, Van Zyl SR, Badri M, Pai M. T-cell interferon- release assays for the rapid immunodiagnosis of tuberculosis: Clinical utility in high-burden vs. low-burden settings. Curr Opin Pulm Med. 2009;15(3):188-200.
33. Mandalakas AM, Detjen AK, Hesseling AC, Benedetti A, Menzies D. Interferon-gamma release assays and childhood tuberculosis: systematic review and meta-analysis. Int J Tuberc Lung Dis. 2011;15(8):1018-32.

34. Adetifa IM, Ota MO, Jeffries DJ, Hammond A, Lugos MD, Donkor S, Patrick $\mathrm{O}$, Adegbola RA, Hill PC. Commercial interferon gamma release assays compared to the tuberculin skin test for diagnosis of latent Mycobacterium tuberculosis infection in childhood contacts in the Gambia. Pediatr Infect Dis J. 2010;29(5):439-43.

35. Hesseling AC, Schaaf HS, Gie RP, Starke JR, Beyers N. A critical review of diagnostic approaches used in the diagnosis of childhood tuberculosis. The International Journal of Tuberculosis and Lung Disease. 2002;6(12):1038-45.

36. TB CARE I. International Standards for Tuberculosis Care. 3rd ed. The Hague: TB CARE I; 2014

37. Jackson C, Southern J, Whitworth H, Scott M, Tsou C-Y, Sridhar S, Nikolayevskyy V, Lipman M, Sitch A, Deeks J, Griffiths C, Drobniewski F, Lalvani A, Abubakar I. S57 Diabetes and latent tuberculosis infection: nested case-control study within the PREDICT cohort. Thorax. 2013;68 Suppl 3:A31-2.

38. Sollai S, Galli L, De Martino M, Chiappini E. Systematic review and metaanalysis on the utility of Interferon-gamma release assays for the diagnosis of Mycobacterium tuberculosis infection in children: a 2013 update. BMC Infect Dis. 2014;14(1):S6.

\section{Submit your next manuscript to BioMed Central and we will help you at every step:}

- We accept pre-submission inquiries

- Our selector tool helps you to find the most relevant journal

- We provide round the clock customer support

- Convenient online submission

- Thorough peer review

- Inclusion in PubMed and all major indexing services

- Maximum visibility for your research

Submit your manuscript at www.biomedcentral.com/submit
C Biomed Central 